\title{
ene
}

\section{ANÁLISIS DE LA INTERVENCIÓN DE ENFERMERÍA CON UNA POBLACIÓN ADULTA TRABAJADORA}

\section{ANALYSIS OF THE NURSING INTERVENTION WITH A WORKING ADULT POPULATION}

Daniel Martínez Esquivel, Mónica Pilar Muñoz Jiménez, Patsy Quesada Carballo,

Yerlin Quesada Rodríguez

Universidad de Costa Rica

Martínez Esquivel, D., Muñoz Jiménez, M., Quesada Carballo, P., \& Quesada Rodríguez, Y. (2020). ANÁLISIS DE LA INTERVENCIÓN DE ENFERMERÍA CON UNA POBLACIÓN TRABAJADORA. Revista Ene De Enfermería, 14(3). Consultado de http:// www.ene-enfermeria.org/ojs/index.php/ENE/article/view/997 


\section{Resumen}

Introducción. La Enfermería es una ciencia que se ha posicionado en diferentes ámbitos, como el escenario laboral, a partir de la prestación de cuidados fundamentados en teorías propias, el Proceso de Enfermería y el uso de lenguaje estandarizado; generando resultados positivos en la vida de las personas desde la promoción de la salud. Objetivo. Analizar la intervención de Enfermería con un grupo de adultos trabajadores. Materiales y método. Caso clínico basado en el estudio de caso que se desarrolla a partir de las etapas del Proceso de Enfermería y el análisis secundario de datos. Resultados. Los patrones funcionales alterados o con riesgo de alteración demostrados en el grupo de adultos trabajaros fueron: nutricionalmetabólico, actividad-ejercicio, percepción-manejo de la salud, afrontamientotolerancia del estrés y sueño-reposo. Discusión. La Teoría General de Enfermería permitió dirigir la intervención de Enfermería a partir de su propuesta de autocuidado, déficit de autocuidado y los sistemas enfermeros. Conclusión. La intervención de Enfermería en el ámbito laboral se enfoca en la promoción y la prevención fundamentada en el sistema enfermero de apoyo educativo.

\section{Palabras clave: Autocuidado;} Enfermería; Proceso de Enfermería; Enfermería del Trabajo. 


\section{Abstract}

Introduction. Nursing is a science that has positioned itself in different areas, such as the workplace, from the provision of care based on own theories, the Nursing Process and the use of standardized language; generating positive results in the lives of people from the promotion of health. Aim. Analyze the nursing intervention with a group of working adults. Methods. Clinical case based on the case study that develops from the stages of the Nursing Process and the secondary analysis of data. Results. The functional patterns altered or with the risk of alteration demonstrated in the group of working adults were: nutritional-metabolic, Activity-exercise, perception-management of health, coping-tolerance of stress and sleep-rest. Discussion. The General Theory of Nursing allowed directing the nursing intervention based on its self-care proposal, self-care deficit, and the nursing systems. Conclusion. Nursing intervention in the workplace is focused on promotion and prevention based on the educational support nurse system.

Key Words: Nursing; Nursing Process; Occupational Health Nursing; Self Care. 


\section{INTRODUCCIÓN}

El presente artículo expone un caso clínico referente a una intervención desarrollada en el 2019 con un grupo de personas adultas trabajadoras enmarcada en el curso "EE-0413 Módulo de Intervención de Enfermería con la adultez sana", perteneciente al plan de estudios de la carrera de Licenciatura de Enfermería de la Universidad de Costa Rica (UCR).

El mismo se fundamentó en la aplicación teórica, el desarrollo del Proceso de Enfermería (PE) y el uso de lenguaje estandarizado de Enfermería permitiendo fortalecer el ser, el saber y el hacer de la profesión a partir de la prestación de cuidados de forma racional, crítica y sistémica.

En este sentido, la Enfermería a través de la historia ha avanzado de ser una práctica empírica a una profesión que utiliza modelos o teorías que se encarga de dirigir la atención para comprender a las personas en su ciclo de vida, no solo prodigando cuidados dependientes $\mathrm{o}$ interdependientes, sino también cuidados independientes en los diferentes escenarios donde se desarroIla (1).

Según Raile y Marriner, "Las teorías de Enfermería se han convertido en un tema importante, estimulando el crecimiento notable y la inmensa expansión de la educación y la bibliografía de la Enfermería. Desarrollar un conocimiento enfermero sobre el que pudiera basarse la práctica fue un objetivo principal propuesto por las líderes de la profesión enfermera en el siglo $X X$, porque buscaban mejorar la práctica y obtener el reconocimiento de la Enfermería como una profesión" (2).

Gracias a esto, el conocimiento de la profesión permitió justificar las acciones que se realizan dentro del trabajo enfermero, otorgándole autonomía en su ejercicio profesional al brindar cuidados en el proceso de salud de las personas.

Este caso clínico se enmarcó desde el concepto de salud entendida como un estado dinámico en el ciclo vital, que implica una adaptación permanente al estrés en el entorno interno y externo mediante el uso óptimo de los recursos de cada uno para conseguir el máximo rendimiento para la vida diaria (3). También como un derecho humano fundamental, donde todos y todas deben gozar del mayor grado posible sin ningún tipo de prejuicio o discriminación (4). Además, como un estado de bienestar determinado por múltiples ámbitos como sociales, económicos, físicos, políticos, desarrollo sociohistórico y laborales (5).

Según Vélez (6) los determinantes de la salud se entienden como las condiciones sociales en que las personas vi- 
ven, trabajan y tienen un impacto en la salud. Para el desarrollo de la intervención fue clave comprender el desarrollo sociohistórico y el trabajo como determinantes de la salud debido a que la experiencia los conjugó para reflejar las necesidades atendidas de la población y a los que Enfermería debió ser sensible para posicionarse de manera empática y compasiva promulgando un cuidado humanizado (7).

Por un lado, la adultez se entiende como una etapa de la vida que está moldeada por la sociedad y la cultura. Se refiere a la existencia de una persona quien, desde diferentes puntos de vista, se encuentra orgánicamente desarrollada, podría gozar de independencia y solvencia; es capaz de responsabilizarse por sus actos y realizar un aporte al medio social en el que se desarrolla, al tiempo que presenta cualidades psicológicas con independencia de criterio $(8,9)$.

Por otro lado, el trabajo para la población adulta temprana, media y tardía implica un aspecto que influye en la salud propiamente dicha, en la calidad de vida, en la productividad y en la motivación laboral. Un lugar de trabajo saludable promueve una buena salud, que es un recurso primordial para el desarrollo personal así como una dimensión importante de los ambientes laborales (10).
En este sentido, las enfermeras, a través de la promoción de la salud, promueven entornos laborales saludables fomentando el desarrollo personal, familiar y social en la adultez. La promoción ha sido definida como el proceso de capacitar a las personas para que aumenten el control sobre su salud y para que la mejoren. Esta se consigue a través de tres mecanismos intrínsecos de las personas.

Según lo planteado por la Organización Panamericana de la Salud (OPS), estos son: 1. El autocuidado, es decir, las decisiones y acciones que la persona lleva a cabo en beneficio de su propia salud. 2. La ayuda mutua o acciones que las personas realizan para ayudarse unas a otras de acuerdo a la situación que estén viviendo. 3. Los entornos sanos o creación de las condiciones ambientales que favorecen la salud (11).

Por lo tanto, a partir de estos mecanismos y siguiendo el proceso de cuidado, la Enfermería apoya el cumplimiento de las metas definidas por empleadores y trabajadores, logrando centros de trabajo con condiciones dirigidas hacia el bienestar, las buenas relaciones personales, la organización positiva y la salud emocional (10).

De esta forma, se comprende la trascendencia de la intervención de Enfermería en el ámbito laboral con el fin 
de potenciar la salud en el trabajo siendo de gran importancia para la satisfacción de las necesidades de los trabajadores que en ocasiones se ve perjudicada por las condiciones de trabajo, la sobrecarga, el sedentarismo, entre otros factores de riesgo.

Así se evidencia que las funciones del profesional en Enfermería van más allá del área hospitalaria, posicionándose en otras como parte del proceso de atención integral (9). Debido a lo anterior, este documento tiene como objetivo analizar la intervención de Enfermería con una población adulta trabajador.

\section{Material y MÉtodo}

El presente caso clínico se enmarcó en la metodología de estudio de caso ya que buscó comprender un fenómeno particular dentro de su entorno específico, siendo así la salud de un grupo de adultos en su lugar de trabajo (12). Además, se realiza un análisis secundario de los datos obtenidos en la experiencia de intervención de Enfermería en el escenario propiamente dicho (13).

Su desarrollo se fundamentó en el PE. Este se define como el sistema de la práctica de Enfermería, en el sentido de que proporciona el mecanismo por el que el profesional utiliza sus opiniones, sus conocimientos y sus habilidades para diagnosticar y tratar la respuesta del cliente a los problemas reales o potenciales de la salud (14).

Dicho proceso se asemeja al mé todo científico, ya que a partir del contexto, datos y experiencias se valora una situación de salud. A partir de ella se plantea una problemática que se diagnostica realizando una revisión del tema basado en la evidencia científica. Se formula una meta que se asemeja a la hipótesis, se realiza una planeación y ejecución de acciones. Por último, se analizan y evalúan los resultados para finalmente registrarlos (14).

EI PE tuvo una duración de 8 semanas distribuidas de la siguiente manera:

Semanas 1-4. Para la etapa de valoración, se aplicó la entrevista de Enfermería basada en los patrones funcionales de Marjory Gordon, además la valoración física, antropométrica, de signos vitales, de agudeza visual, de audiometría y de medida de la circunferencia abdominal. Se trabajó con un grupo de 12 adultos trabajadores inmersos en un escenario laboral. A nivel grupal se utilizó la estrategia "Buzón de necesidades", para la identificación de necesidades y elementos inmersos en este escenario mediante la respuesta a diversas preguntas anónimas. 
Semanas 4-5. En la fase diagnóstica, se realizó un análisis crítico y reflexivo de la información obtenida durante la valoración. A partir de ello, se determinaron los diagnósticos haciendo uso del lenguaje estandarizado NANDA, y posterior definición de NIC y NOC para el desarrollo de planes de cuidados haciendo uso de la plataforma NNNConsult. Incluso se determinó la puntuación inicial de los indicadores NOC elegidos.

Además, se plantearon 3 sesiones grupales, las cuales se realizaron con el fin de abordar las temáticas propuestas por los mismos funcionarios según el método diagnóstico grupal utilizado.

Semanas 5-7. En la etapa de ejecución, se expuso y negoció con la persona el plan de cuidados. Posteriormente, se implementaron las estrategias individuales para la atención de los patrones alterados y en riesgo. También, se desarrollaron las sesiones grupales sobre los temas: higiene del sueño y manejo del estrés; actividad física y pausa activa; e hidratación y alimentación saludable.

Semanas 7-8. Finalmente, en la etapa de evaluación se estableció la puntuación final según los indicadores NOC. Se reconocieron los cambios y los objetivos logrados por las personas me- diante la realización de las actividades propuestas en la implementación.

\section{Consideraciones éticas}

Se tomaron en cuenta principios de Bioética siguiendo lo estipulado en el Reglamento ético científico de la Universidad de Costa Rica para las investigaciones en las que participan seres humanos (15).

Principio de Autonomía, cada persona tuvo derecho de brindar su consentimiento para participar. Se utilizó el formulario de Consentimiento Informado propuesto por el Comité Ético Científico (CEC) de la UCR ${ }^{(15) .}$

Principio de Beneficencia, a cada participante se le brindó la información científica pertinente y verídica, de acuerdo a los diferentes diagnósticos encontrados, así como en las sesiones de grupo (15).

Principio de No Maleficencia, no se puso en riesgo la integridad física ni mental de ninguno de los participantes y las facilitadoras tuvieron la formación práctica y teórica para intervenir de manera adecuada a los funcionarios participantes (15).

Principio de Justicia, las facilitadoras intervinieron de manera integral y en igualdad de condiciones para todos los integrantes de esta (15). 


\section{REsultados}

\section{DESCRIPCIÓN DEL CASO}

Funcionarias y funcionarios de una institución pública, con edades que los ubicaban en las etapas de la adultez temprana y media, con nivel educativo universitario completo e incompleto en áreas como la psicología, la administración y el secretariado.

\section{VALORACIÓN}

Como parte de la intervención de Enfermería en el ámbito laboral, se realizó la valoración integral a 12 adultos. Por un lado, según la entrevista de Enfermería, se identificaron patrones funcionales con alteración o riesgo de alteración. EI criterio utilizado para definirlos se realizó con base en lo propuesto por Álvarez, Castillo, Fernández y Muñoz (16). Estos se ven representados en el Cuadro 1 y en el Cuadro 2 respectivamente.

En el cuadro anterior, se observa que dentro de los patrones alterados que con mayor frecuencia se demuestran dentro de esta población trabajadora, se encuentra el patrón 2 Nutricional-Metabólico con una presencia del $58.33 \%$ en la población total, el patrón 4 ActividadEjercicio que se presentó en un $50 \%$ del total de participantes y el patrón 1 Per-
cepción-Manejo de la salud presente en un $41.66 \%$.

Además, en el cuadro anterior se refleja que los riesgos de alteración con mayor frecuencia se encontraron en los funcionarios fueron el patrón 10 Afrontamiento-Tolerancia al estrés que representa el $25 \%$ del grupo, el patrón $2 \mathrm{Nu}$ tricional-Metabólico con un $16.66 \%$ del total y el patrón 5 Sueño-Reposo igualmente con un $16.66 \%$.

Por otro lado, los resultados a nivel de valoración física más relevantes indicaron que las condiciones de higiene y el aspecto personal fueron adecuados, sin embargo algunos presentaron problemas de halitosis y mala higiene en sus piezas dentales. No presentaron alteraciones sobresalientes en cabeza, tórax, espalda y miembros superiores e inferiores. En los resultados antropométricos, el $25 \%$ de las 12 personas evaluadas presentaron sobrepeso (IMC: > $25 \mathrm{~kg} / \mathrm{m} 2)$ (17), y el $16.66 \%$ obesidad (IMC: $>30 \mathrm{~kg} / \mathrm{m} 2)$ (17,18). Esto según el cálculo del Índice de Masa Corporal (IMC) tomando en cuenta el peso y la talla de los participantes.

\section{DIAGNÓSTICO}

A partir del juicio clínico, se definieron los diagnósticos de Enfermería utilizando la taxonomía de NANDA. Al analizarlos, los principales diagnósticos 
encontrados se presentan en el $\underline{\text { Cuadro }}$

3.

En el caso de los diagnósticos de Enfermería según la taxonomía NANDA, se identificó el [00168] Estilo de vida sedentario, el cual representa un $41.66 \%$ en la población en estudio. Presentó como características definitorias: actividad física diaria promedio es menor a la recomendada por sexo y edad; y motivación insuficiente para la realización de la misma.

Respecto al diagnóstico [00233] Sobrepeso, cobra importancia con un $25 \%$ de presencia en los participantes y características definitorias tales como un IMC mayor a $25 \mathrm{~kg} / \mathrm{m} 2$ (17, 18). Por último, dentro de los diagnósticos principalmente encontrados se presenta el [00177] Estrés por sobrecarga, igualmente con un $25 \%$ de la totalidad de la población y con características definitorias como tensión e impacto negativo del estrés como por ejemplo: síntomas físicos, sufrimiento psicológico, sensación de estar enfermo $(17,18)$.

Por otra parte, mediante los datos recolectados a partir de las respuestas obtenidas en el "Buzón de necesidades", se destacan los siguientes diagnósticos: [00163] Disposición para mejorar la nutrición, [00165] Disposición para mejorar el sueño, [00168] Estilo de vida sedentario, [00177] Estrés por sobrecarga (17).

\section{Planificación}

Para la etapa de planificación se utilizó el lenguaje taxonómico, del cual se seleccionaron los NOC, los cuales permiten identificar, denominar, validar y clasificar resultados e indicadores del usuario susceptibles para ser alcanzados mediante la intervención de Enfermería $(19,20)$. En el Cuadro 4 se presenta un resumen de los NOC utilizados.

Los NOC más destacados a partir de los diagnósticos encontrados se representan en el cuadro 4, en el cual se denota motivación, nivel de estrés, participación en el ejercicio, conocimiento: vida saludable y dieta saludable. Estos se plasmaron en los planes de Enfermería a través de la elección de NIC y actividades orientadas a generar un cambio en los indicadores referentes.

\section{EJECUCIÓN}

Se establecieron NIC, los cuales ofrecen un lenguaje consensuado y codificado de intervenciones de Enfermería desplegados de los diagnósticos y NOC $(18,20)$. En el Cuadro 5 se presentan los NIC utilizados.

Dentro de los NIC utilizados con mayor frecuencia se encuentran principalmente mejorar el afrontamiento y modificar la conducta en un tercio de la población; asesorar y fomentar el ejerci- 
cio en un cuarto del total de los participantes.

En cuanto a las estrategias que se utilizaron para la ejecución en la atención individual están el asesoramiento personalizado en temas relacionados con la nutrición, el control del estrés y el fomento de la actividad física. Se motivó a cada persona al cambio de conductas referentes a su estado de salud, la proposición de sus propios objetivos, tomar responsabilidad de su autocuidado y ejercer cambios en aspectos relacionados a hábitos de vida o inicio de acciones que beneficien su salud.

En la atención grupal se llevaron a cabo estrategias que promovieran la participación del grupo, tales como espacios para que opinen sobre los temas tratados en actividades expositivas. Con el fin de establecer un vínculo con las facilitadoras, se realizaron dinámicas de actividad física y pausa activa. Se entregó material educativo digital e impreso sobre las temáticas tratadas y se realizó una actividad evaluativa de los conocimientos adquiridos así como la importancia de la sesión impartida.

También se realizaron intervenciones de manera indirecta como un recordatorio constante por medio de infografías sobre temas como higiene bucal, lavado de manos y consumo adecuado de agua. Estas se colocaron en espacios visibles y frecuentados, como el baño y la cocina. Esta estrategia permitió accesar a todas las personas, incluso las que no se atendieron individualmente ni en las sesiones grupales.

\section{Evaluación}

En la etapa de evaluación se establecieron los indicadores mostrados en el Cuadro 6. Estos correspondieron a los indicadores presentes en la población con mayor frecuencia, se evaluaron con respecto a la intervención individual y grupal.

Estos indicadores fueron evaluados en función de la escala correspondiente a cada uno según la taxonomía NANDA, NIC y NOC. La escala $m$ corresponde a las puntuaciones de 1-5; donde 1 es Nunca demostrado, 2 es Raramente demostrado, 3 A veces demostrado, 4 Frecuentemente demostrado y 5 Siempre demostrado. La escala $n$ de 1-5; donde 1 es Grave, 2 es Sustancial, 3 Moderado, 4 Leve y 5 Ninguno. Y la escala u de 1-5 donde 1 es Ningún conocimiento, 2 es Conocimiento, 3 Conocimiento moderado, 4 Conocimiento sustancial y 5 Conocimiento extenso (17, 18, 19).

Como se observa en las puntuaciones finales, se obtienen resultados exitosos, los cuales logran llegar o incluso superar la puntuación diana previa- 
mente establecida, lo cual reflejó como el plan y las actividades realizadas en función a fomentar el autocuidado en los participantes alcanzaron el objetivo.

\section{Discusión}

Durante el proceso de atención individual y grupal se tomó como base a la propuesta de Dorothea Orem, la cual etiquetó su teoría del déficit de autocuidado como una teoría general compuesta por las siguientes tres teorías relacionadas: teoría del autocuidado, que describe el porqué y cómo las personas cuidan de sí mismas; teoría de déficit de autocuidado, que describe y explica cómo la Enfermería puede ayudar a las personas; y teoría de sistemas enfermeros, que describe y explica las relaciones que hay que mantener para que se produzca el cuidado enfermero (2).

Además, para aplicar este modelo fue necesario conocer cómo se definen los metaparadigmas con el propósito de crear un marco que dimensionara esta intervención (21). El ser humano se concibe como un organismo biológico y racional; los seres humanos tienen la capacidad de reflexionar sobre sí mismos y su entorno, capacidad para simbolizar lo que experimentan y usar creaciones simbólicas (ideas, palabras) para pensar, comunicarse y guiar los esfuerzos para hacer cosas que son beneficio- sas para sí mismos y para otros (21). En este sentido, se comprendió que la persona tiene las capacidades necesarias para cuidar de su salud y de la de otros; o para adquirirlas en los casos donde no las tiene.

Los cuidados de Enfermería se refieren a la ayuda que se le ofrece al individuo para llevar a cabo y mantener acciones de autocuidado que conserven la salud y la vida. Al mismo tiempo, que le permitan recuperarse de la enfermedad y afrontar las consecuencias de ésta (21). Por lo tanto, la Enfermería se posicionó como una facilitadora del empoderamiento que apoya la recuperación, el mantenimiento y la rehabilitación de la salud de las personas.

En cuanto al entorno, es entendido en este modelo como todos aquellos factores físicos, químicos, biológicos y sociales, ya sean familiares o comunitarios, que pueden influir e interactuar con la persona (21). Lo que permitió comprender que existen múltiples causas que pueden influir en el bienestar personal o grupal teniendo una visión moderna de los factores que influyen en el goce de la salud.

Finalmente, el concepto de salud es definido como un estado cambiante determinado por las características humanas y biológicas (21). Sin embargo, se vislumbró que las características socia- 
les, económicas y políticas en las que viven las personas, igualmente impactan el dinamismo de la salud.

Una vez elaborada la dimensión en la que se desarrolló esta intervención fue importante acercarse al concepto de autocuidado, el cual es clave para entender la teoría que fundamentó las acciones de Enfermería. Orem se refiere al autocuidado como "la práctica de actividades que las personas inician y hacen por sí mismas para el mantenimiento de la vida, la salud y el bienestar" (22). Además, según Orem, el autocuidado no es innato sino que se aprende a lo largo de la vida a través de las relaciones interpersonales y la comunicación en la familia, la escuela y amigos" (23).

El autocuidado va encaminado a la modificación de hábitos higiénicos, alimentarios, tóxicos, del comportamiento sexual entre otros, y a los cambios ambientales dirigidos al ambiente físico (23).

En referencia a esto, en el escenario de intervención se encontraron necesidades grupales e individuales de autocuidado reflejadas en conductas que afectaban o podrían afectar el mantenimiento de la salud. Dichas necesidades estuvieron relacionadas con la higiene del sueño; hábitos alimentarios saludables debido a la alta incidencia de sobrepeso y obesidad encontradas en las va- loraciones; y el manejo del estrés que se abordó mediante la promoción de actividad física y la pausa activa, así como la relajación por medio de la meditación.

El autocuidado tiene su razón de ser, es la acción que tiene un modelo, un desarrollo y, cuando se realiza eficazmente, contribuye de forma específica a la integridad estructural, funcionamiento y desarrollo humano (22). Las intervenciones realizadas, se llevaron a cabo con el fin de direccionar los cambios sobre este eje, mediante el refuerzo de actividades relacionadas al autocuidado.

Los propósitos para obtener los tipos de acción de autocuidado se llaman requisitos de autocuidado (22). Un requisito de autocuidado es un consejo formulado y expresado sobre las acciones que se deben llevar a cabo, ya sea de manera continua o bajo unas circunstancias y condiciones específicas. Los requisitos formulados y expresados, representan los objetivos formalizados del autocuidado. Son las razones por las que se emplea el autocuidado ya que expresan los resultados deseados a través del objetivo del autocuidado (2).

En la institución pública, se deseaba cumplir el objetivo de promover los hábitos saludables en el grupo de adultos mediante las intervenciones realizadas. Dichas actividades tuvieron el propósito de concientizar a los partici- 
pantes sobre temas de salud que satisficieran las necesidades de autocuidado de los mismos según se identificaron en los requisitos universales.

Los objetivos requeridos universalmente que deben alcanzarse mediante el autocuidado o el cuidado dependiente tienen sus orígenes en lo que se conoce y lo que se valida o lo que está en proceso de ser validado sobre la integridad estructural y funcional humana en las diversas etapas del ciclo vital. Orem (2), propone ocho requisitos comunes a todo ser humano:

“1. El mantenimiento de un aporte suficiente de aire.

El mantenimiento de un aporte suficiente de alimentos.

El mantenimiento de un aporte suficiente de agua.

La provisión de cuidado asociado con los procesos de eliminación.

El mantenimiento de un equilibrio entre la actividad y el descanso.

El mantenimiento de un equilibrio entre la interacción social y la soledad.

La prevención de peligros para la vida, el funcionamiento y el bienestar humano.

La promoción del funcionamiento humano y el desarrollo en los grupos sociales de acuerdo con el potencial hu- mano, las limitaciones humanas conocidas y el deseo humano de ser normal" (2).

Uno de los requisitos universales a los que se les dio prioridad en esta intervención fue el mantenimiento de un aporte suficiente de alimentos, debido a la necesidad de educación en temas referentes a la nutrición balanceada, la lectura de etiquetas y la cantidad de porciones diarias requeridas; así como los hábitos alimentarios inadecuados que se identificaron en las valoraciones individuales. Los funcionarios expresaron interés en esta temática, además los datos valorados indicaron que el $41.66 \%$ de los participantes presentaba sobrepeso u obesidad.

Adicional a esto, la información recolectada en la entrevista de Enfermería evidenció la necesidad de priorizar el requisito universal del mantenimiento de un aporte suficiente de agua, ya que la mayoría de los participantes consumía menos de $1 \mathrm{~L}$ de agua diaria relacionado a falta de tiempo, de interés o de conocimiento de los beneficios que conlleva el consumo de la misma. Se dispuso a intervenir individualmente a las personas que lo ameritaban, a nivel grupal se realizó una sesión que incluyó esta temática, importancia, beneficios y situaciones que afectan la salud debido al no consumo de agua. 
Igualmente, los funcionarios mos-

traron necesidad de mantener un equilibrio entre la actividad y el descanso, ya que en su mayoría mantienen un estilo de vida sedentario y altos niveles de estrés por motivos como sobrecarga de trabajo, horarios poco flexibles, vida urbana, cansancio laboral, entre otros. Mediante sesiones grupales se abordaron dichos temas, resultados favorables como la contratación de una instructora de acondicionamiento físico.

El déficit de autocuidado en una persona aparece cuando la demanda es superior a la capacidad del individuo para realizar el autocuidado. Es aquí cuando tiene acción el profesional de Enfermería para compensar la demanda de cuidados (23). Los requisitos descritos anteriormente, mostraron la necesidad de agenciar la capacidad del individuo para afrontarlos a partir de las acciones de Enfermería que lograran satisfacer sus demandas.

La idea central de la teoría del déficit de autocuidado es que las necesidades de las personas que precisan de la Enfermería se asocien a la subjetividad de la madurez y de las personas maduras relativa a las limitaciones de sus acciones relacionadas con su salud o con el cuidado de su salud. Estas limitaciones vuelven a los individuos completa o parcialmente incapaces de cono- cer los requisitos existentes y emergentes para su propio cuidado regulador 0 para el cuidado de las personas que dependen de ellos. El déficit de autocuidado es un término que expresa la relación entre las capacidades de acción de las personas y sus necesidades de cuidado (23).

La Enfermería en este contexto es protagónica ya que la capacidad del profesional se refleja al establecer relaciones interpersonales legítimas para reconocer, actuar y ayudar a las personas implicadas en cubrir las necesidades terapéuticas de autocuidado requeridas. Además, ayuda a regular el desarrollo o el ejercicio de la actividad de autocuidado de la persona por medio del diseño enfermero. El diseño enfermero es una actividad profesional desarrollada tanto antes como después del diagnóstico y la prescripción enfermera. Surge a partir de los juicios prácticos de reflexión sobre las condiciones existentes para sintetizar los elementos de una situación concreta de relaciones ordenadas a unidades operativas de estructura (2).

El objetivo del diseño enfermero se constituye con el fin de ofrecer una guía para alcanzar los resultados necesarios y previstos hacia el logro de las metas establecidas de acuerdo a cada caso mediante el desarrollo de los métodos de ayuda (2). 
Un método de ayuda es una serie secuencial de acciones que resolverá o compensará las limitaciones asociadas a la salud de las personas que se comprometen a realizar acciones para regular su propio funcionamiento y desarrollo, o el de sus dependientes. Las enfermeras utilizan todos los métodos, los seleccionan y los combinan en relación con las necesidades de acción de las personas que reciben tratamiento enfermero $y$ en relación con las limitaciones de acción asociadas con su estado de salud. Los métodos de ayuda son: actuar o hacer por cuenta de otro; guiar y dirigir; ofrecer apoyo físico o psicológico; ofrecer y mantener un entorno que fomente el desarrollo personal; y enseñar (2).

En el caso de esta intervención se hizo uso del método de ayuda de enseñar a otro ya que se ajustó a la dinámica del lugar, siendo la mejor estrategia posible para compensar los requisitos de autocuidado que demandaron atención de Enfermería. Enseñar a otro es un método válido para ayudar a una persona o a un paciente que necesita instrucción para desarrollar conocimientos o habilidades específicas. Es necesario que el ayudante sepa muy bien qué es lo que necesita conocer la persona a la que le va a enseñar (22). Este permitió formar al grupo de funcionarios en el desarrollo de competencias que favorecieran el empoderamiento de su autocuidado.

Sumado a esto, la teoría de los sistemas enfermeros señala que la Enfermería es una acción humana; los sistemas enfermeros son sistemas de acción deliberada, que abarcan las intenciones y las actividades de diagnóstico, la prescripción y la regulación en función de las necesidades terapéuticas para proteger y regular el ejercicio o desarrollo de la actividad de autocuidado (2).

Los sistemas enfermeros pueden ser elaborados para personas y para familias $u$ otros grupos multipersonales (2) como en el caso de esta intervención. Cuando existe el déficit de autocuidado, los profesionales de Enfermería pueden compensarlo por medio de algunos de los sistemas enfermeros, los cuales pueden ser:

\section{Sistema parcialmente compen-} sador: cuando el individuo presenta algunas necesidades de autocuidado por parte de la enfermera, bien por motivos de limitación o incapacidad.

\section{Sistema totalmente compensa} dor: cuando el individuo no puede realizar ninguna actividad de autocuidado. Implica una dependencia total.

Sistema de apoyo educativo: cuando el individuo necesita orientación y enseñanza para llevar a cabo el autocuidado; por consiguiente la enfer- 
mera ayuda al paciente con sus consejos, apoyo y enseñanzas de las medidas que sean necesarias para que el individuo sea capaz de realizar su autocuidado (25).

El tipo de sistema determina el grado de participación del individuo para ejecutar su autocuidado, regularlo o rechazar el cuidado terapéutico. Una persona o grupo puede pasar de un sistema de Enfermería a otro en cualquier circunstancia, o puede activar varios sistemas al mismo tiempo (25).

Por este motivo, al identificar la agencia de autocuidado del grupo de funcionarios, sus capacidades y sus necesidades, se trabajó en base al sistema de apoyo educativo, el cual fomentó la práctica de acciones en favor de la salud tanto física como mental por medio de intervenciones efectivas enfocadas en el método de ayuda basado en la enseñanza. El sistema de apoyo educativo fue el pilar de la intervención, ya que todas las actividades grupales e individuales se realizaron bajo esta línea debido a las necesidades presentes en esta población.

Por su parte, según Orem (22), el profesional de Enfermería debe ser capaz de lograr que el individuo participe de forma activa en la planificación e implementación de su propia atención de salud, teniendo en cuenta los factores básicos condicionantes del autocuidado los cuales de acuerdo a Naranjo, Concepción y Rodríguez (26), hacen referencia a los factores que condicionan o alteran el valor de la demanda de autocuidado terapéutico y/o actividad de autocuidado de un individuo en momentos concretos y bajo circunstancias específicas.

Entre estos se encuentran la edad, el sexo, el estado de desarrollo, estado de salud y los conocimientos que poseen de ésta, la pertenencia al grupo social, factores familiares, y los hábitos del medio sociocultural, disponibilidad de recursos, la falta de habilidades y hábitos, el concepto de sí mismo y la madurez de la persona (22, 26).

En este caso, los participantes se caracterizan por ser adultos, con una situación de salud favorable, comprometidos e interesados en realizar un cambio en sus hábitos de vida para mejorar. Los mismos expresan que estos espacios son efectivos ya que les permiten compartir opiniones e ideas y aprender de manera sustancial sobre estos temas de salud; y realizar autoanálisis introspectivos sobre su propio estado de salud físico y mental.

De esta manera, la intervención de Enfermería, además de enfocarse en el apoyo educativo, busca que los participantes identifiquen sus necesidades y 
logren planificar una acción en pro al cambio de actitudes negativas y hábitos perjudiciales, y al fomento del autocuidado.

En la intervención realizada, el sistema de apoyo educativo se individualizó y concretó en función de cada persona o situación, a través de una valoración que facilitó la determinación de los principales diagnósticos, las intervenciones y las actividades específicas a realizar, así como la evaluación de los resultados mediante el uso de la clasificación taxonómica de NANDA, NOC, NIC (25).

\section{CONCLUSIONES}

La intervención de Enfermería dentro del ambiente laboral está enfocada a la promoción de la salud y la prevención de la enfermedad mediante el apoyo educativo.

Las necesidades de la población adulta sana van en dirección a estilos de vida saludables, relacionados con aspectos de nutrición, manejo del estrés y equilibrio entre el trabajo y la actividad física.

El abordaje que se realizó se fundamentó en el sistema de apoyo educativo, tratando el autocuidado, dónde se pretende que los individuos aprendan a adaptarse a las diferentes situaciones, con las herramientas que le provee el personal de Enfermería y sus propias capacidades para mejorar su agencia de autocuidado.

Por medio de las sesiones individuales, se logró crear un espacio de confianza y respeto, mediante el cual se trataron los temas más individualizados a cada situación personal, y a través del planeamiento de actividades e implementación se logró cumplir con los objetivos propuestos, al resultar exitosas las evaluaciones realizadas de los indicadores.

Mediante las sesiones grupales se logró que los participantes, a través de los espacios educativos, así como los de expresión y compartir de opiniones, tomaran conciencia sobre el tema de autocuidado. 


\section{BIBLIOGRAFÍA}

1. Reyes E. Fundamentos de Enfermería. Ciencia, Metodología y Tecnología. [Internet] 2da Edición. México DF: El Manual Moderno; 2015. [citado 6 mayo 2019] Disponible en https://books.google.es/ b $\quad 0 \quad$ o $\quad k \quad$ s ? $\mathrm{hl}=e s \& \mathrm{r}=\& \mathrm{id}=\mathrm{zO} 4 \mathrm{VCgAAQBAJ} \&$ oi=fnd\&pg $=\mathrm{PT} 23$ $\& d q=$ info:cZ7MWk1ShkYJ:scholar.google.com/ \&ots $=$ PLd6nOAb1T\&sig=qXk6qT4L5uKocUks-

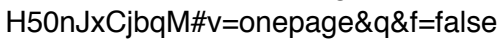

2. Raile M, Marriner A. Modelos y Teorías en Enfermería. 7ma Edición. Barcelona: Elsevier España: 2011.

3. King I. Enfermería como profesión. Filosofía, principios y objetivos. México: Editorial Limusa S.A.; 1984.

4. Organización Mundial de la Salud. Declaración de Astaná. Astaná: WHO/UNICEF; 2018.

5. Biedma L, Cerrillo J, Serrano R. Una aproximación a la cultura sanitaria de los residentes andaluces. Fundación INDEX. [Internet] 2009 [citado 6 de mayo 2019]; N. 2: 1-10.

6. Vélez C, Escobar M, Pico M. Determinantes sociales de la salud y el trabajo informal. Rev Costarr Salud Pública. [Internet] 2013 [citado 6 de mayo 2019]; 22(2): 156-162. Disponible en http://www.scielo.sa.cr/pdf/rcsp/v22n2/art11v22n2.pdf

7. Ramírez N. Abordaje conceptual del cuidado para mejorar la práctica de Enfermería. Enfermería en Costa Rica. 2016;34(11).

8. Papalia D, Feldman R, Martorell G. Desarrollo Humano. 13 Edición. Ciudad de México: McGrawHill/Interamericana Editores; 2017.

9. Alpízar W. Hacia la construcción del concepto integral de adultez. Revista Enfermería Actual en Costa Rica. [Internet] 2005 [citado 7 de mayo 2019]; 10: 1-14. Disponible en https://revistas.ucr.ac.cr/index.php/enfermeria/article/download/10704/10096/

10.Barrios S, Paravic T. Promoción de la salud y un entorno laboral saludable. Rev. Latino-am Enfermagem. [Internet] 2006 [citado 7 de mayo 2019]; 14 (1): 136-141. Disponible en http://www.scielo.br/ pdf/rlae/v14n1/v14n1a19

11.Giraldo A, Toro M, Macías A, Valencia C, Palacio S. La Promoción De La Salud Como Estrategia Para El Fomento De Estilos De Vida Saludables. Revista Hacia la Promoción de la Salud [Internet]. 2010;15(1):1

12. do Prado ML, de Souza ML, Monticelli M, Cometto MC, Gómez PF. Investigación cualitativa en Enfermería. Metodología y didáctica. Washington D.C.: OPS; 2013.

13.Polit, D., \& Tatano , C. Investigación en Enfermería: Fundamentos para el uso de la evidencia en la practica de la Enfermería (Novena ed.). Barcelona: Wolters Kluwer; 2018.

14.Reina G., NC. El Proceso de Enfermería: instrumento para el cuidado. Umbral Científico [Internet]. 2010;(17):18-23. Disponible en https://www.redalyc. org/articulo.oa?id=30421294003

15.Consejo Universitario. Reglamento ético científico de la Universidad de Costa Rica para las investigaciones en las que participan seres humanos [Internet]. San José, Costa Rica: Universidad de Costa Rica; 2000 [citado 12 de mayo de 2019]. Disponible en: http://www.cu.ucr.ac.cr/normativ/ etico_cientifico.pdf

16.Álvarez J, Castillo F, Fernández D, Muñoz M. Manual de Valoración de Patrones Funcionales. [Internet] Asturias; 2011. [citado 7 de mayo 2019] Disponible en https://seapaonline.org/UserFiles/ File/Ayuda\%20en\%20consulta/MANUAL\%20VALORACION\%20NOV\%202010.pdf

17.NANDA International. Nursing diagnoses. Definitions and classification 2018-2020. 11.a ed. Nueva York: Thieme; 2018.

18. Moorhead S, Swanson E, Johnson M, Maas M. Clasificación de Resultados de Enfermería (NOC). Medición de resultados de salud. Sexta Edición. Barcelona: Elsevier; 2018.

19. Butcher H, Bulechek G, Dochterman J, Wagner C. Clasificación de Intervenciones de Enfermería (NIC). Séptima Edición. Barcelona: Elsevier; 2018

20.Tirado G, Hueso C, Cuevas M, Montoya R, Bonill C, Schmidt J. Como escribir un caso clínico en Enfermería utilizando Taxonomía NANDA, NOC, NIC. Index Enferm. [Internet] 2011; 20(2). Disponible en http://scielo.isciii.es/scielo.php? script=sci_arttext\&pid=S1132-12962011000100023

21. Navarro Y, Castro M. Modelo de Dorothea Orem aplicado a un grupo comunitario a través del proceso de enfermería. Enferm. glob. [Internet]. 2010 Jun [citado 2019 Mayo 13] ; (19). Disponible en: http://scielo.isciii.es/scielo.php? script=sci_arttext\&pid=S1695-6141201000020000 $4 \& \operatorname{lng}=\mathrm{es}$.

22.Orem, D. Normas prácticas en enfermería. Madrid: Ediciones Pirámide; 1983.

23. Rivero N, González M, González M, Núñez M, Hornedo I, Rojas M. Valoración ética del modelo de Dorothea Orem. Rev haban cienc méd [Internet]. 2007 Sep [citado 2019 Mayo 08] ;6 (3). Disponible en: http://scielo.sld.cu/scielo.php? script =sci_arttext\&pid=S1729-519X2007000300012\&Ing=es.

24.Rojas J, Zubizarreta M. Reflexiones del estilo de vida y vigencia del autocuidado en la atención primaria de salud. Rev Cubana Enfermer [Internet]. 2007 Mar [citado 2019 Mayo 08] ; 23(1). Disponible en: http://scielo.sld.cu/scie$\mathrm{l} \quad \mathrm{l} \quad \mathrm{p} \quad \mathrm{h} \quad \mathrm{p}$ ? 
script=sci_arttext\&pid=S0864-0319200700010000 $7 \& \operatorname{lng}=\mathrm{es}$.

25.Prado L, González M, Paz N, Romero K. La teoría Déficit de autocuidado: Dorothea Orem punto de partida para calidad en la atención. Rev. Med. Electron. [Internet]. 2014 Dic [citado 2019 Mayo 08] ; 36(6): 835-845. Disponible en: http://scielo.sld.cu/scielo.php?script=sci_arttext\&pid=S1684$18242014000600004 \&$ Ing=es.

26. Naranjo Y, Concepción J, Rodríguez M. La teoría Déficit de autocuidado: Dorothea Elizabeth Orem. Gac Méd Espirit [Internet]. 2017 Dic [citado 2019 Mayo 12] ; 19(3): 89-100. Disponible en: http:// s c i e I o. s I d. cu / s c i e I o. ph p ?
script=sci_arttext\&pid=S1608-8921201700030000 $9 \& \operatorname{lng}=\mathrm{es}$

27. Wynants L, Van Calster B. Bonten MM.J., Collins, GS, Debray TPA, De Vos M, et al. (2020). Prediction models for diagnosis and prognosis of COVID-19 infection: Systematic review and critical appraisal. The BMJ, 369:m1328. DOI: 10.1136/ bmj.m1328.

\section{CUADROS Y TABLAS}

Cuadro 1. Patrones funcionales alterados en el grupo de adultos trabajadores.

\begin{tabular}{|l|c|c|}
\hline \multirow{2}{*}{ Patrón alterado } & \multicolumn{2}{c|}{ Cantidad } \\
\cline { 2 - 3 } & Número de personas & \\
\hline Nutricional - Metabólico & 7 & $58.33 \%$ \\
\hline Actividad - Ejercicio & 6 & $50 \%$ \\
\hline Percepción - Manejo de la Salud & 5 & $41.66 \%$ \\
\hline
\end{tabular}

Cuadro 2. Patrones con riesgo de alteración en el grupo de adultos trabajadores.

\begin{tabular}{|l|c|c|}
\hline \multicolumn{1}{|c|}{ Patrón con riesgo de alteración } & \multicolumn{2}{c|}{ Cantidad } \\
\cline { 2 - 3 } & Número de persona & \\
\hline Afrontamiento - Tolerancia del Estrés & 3 & $25 \%$ \\
\hline Nutricional - Metabólico & 2 & $16.66 \%$ \\
\hline Sueño - Reposo & & $16.66 \%$ \\
\hline
\end{tabular}


MARTíneZ-ESQUiVel - Muñoz-JimÉneZ - QUESADA-CARBALIO ANÁLISIS DE LA INTERVENCIÓN DE ENEERMERÍA CON UNA POBLACIÓN ADULTA TRABAJADORA

Cuadro 3. Diagnósticos de Enfermería principales presentes en el grupo de adultos trabajadores.

\begin{tabular}{|l|c|c|}
\hline \multicolumn{1}{|c|}{ Diagnóstico de Enfermería } & \multicolumn{2}{c|}{ Cantidad } \\
\cline { 2 - 3 } & Número de personas & \multicolumn{2}{c|}{} \\
\hline [00168] Estilo de vida sedentario & 5 & $21.66 \%$ \\
\hline [00233] Sobrepeso & 3 & $25 \%$ \\
\hline [00177] Estrés por sobrecarga & 3 & \\
\hline
\end{tabular}

Cuadro 4. NOC utilizados en el grupo de adultos trabajadores.

\begin{tabular}{|l|c|c|}
\hline \multicolumn{2}{|c|}{ NOC } & \multicolumn{2}{c|}{ Cantidad } \\
\cline { 2 - 3 } & Número de personas & \multicolumn{2}{c|}{} \\
\hline [1209] Motivación & 4 & $33.33 \%$ \\
\hline [1212] Nivel de estrés & 3 & $25 \%$ \\
\hline [1633] Participación en el ejercicio & 2 & $16.66 \%$ \\
\hline [1855] Conocimiento: Estilo de vida saludable & 2 & $16.66 \%$ \\
\hline [1854] Conocimiento: dieta saludable & 2 & $16.66 \%$ \\
\hline
\end{tabular}

Cuadro 5. NIC utilizados en el grupo de adultos trabajadores.

\begin{tabular}{|l|c|c|}
\hline \multicolumn{2}{|c|}{ NIC } & \multicolumn{2}{c|}{ Cantidad } \\
\cline { 2 - 4 } & Número de personas & \% \\
\hline$[5230]$ Mejorar el afrontamiento & 4 & $33.33 \%$ \\
\hline$[4360]$ Modificación de la conducta & 4 & $33.33 \%$ \\
\hline$[5240]$ Asesoramiento & 3 & $25 \%$ \\
\hline [0200] Fomento del ejercicio & 3 & $25 \%$ \\
\hline
\end{tabular}


MARTíneZ-ESQUiVel - Muñoz-JimÉneZ - QUESADA-CARBALIO ANÁLISIS DE LA INTERVENCIÓN DE ENFERMERÍA CON UNA POBLACIÓN ADULTA TRABAJADORA

Cuadro 6. Evaluación de los indicadores utilizados en el grupo de adultos trabajadores.

\begin{tabular}{|c|c|c|c|c|c|c|}
\hline NOC & Indicadores & Escala & $\begin{array}{l}\text { Puntuación } \\
\text { Inicial }\end{array}$ & $\begin{array}{c}\text { Puntuación } \\
\text { Diana }\end{array}$ & $\begin{array}{c}\text { Puntuación } \\
\text { Final }\end{array}$ & Tiempo \\
\hline \multirow[t]{2}{*}{ [1209] } & $\begin{array}{l}\text { [120902] Desarrolla un } \\
\text { plan de acción. }\end{array}$ & \multirow[t]{2}{*}{ Escala } & 2 & 5 & 5 & 2 semanas \\
\hline & $\begin{array}{l}\text { [120915] Expresa } \\
\text { intención de actuar. }\end{array}$ & & 2 & 4 & 4 & 2 semanas \\
\hline \multirow{2}{*}{$\begin{array}{l}\text { [1633] } \\
\text { Participación } \\
\text { en el } \\
\text { ejercicio. }\end{array}$} & $\begin{array}{l}\text { [163303] Establece } \\
\text { objetivos realistas a corto } \\
\text { plazo. }\end{array}$ & \multirow[t]{2}{*}{ Escala } & 3 & 4 & 4 & 2 semanas \\
\hline & $\begin{array}{l}\text { [163307] Equilibra la rutina } \\
\text { de vida para incluir el } \\
\text { ejercicio. }\end{array}$ & & 2 & 4 & 4 & 2 semanas \\
\hline \multirow[t]{2}{*}{ [1212] Nivel } & $\begin{array}{l}\text { [121214] Trastornos del } \\
\text { sueño. }\end{array}$ & \multirow[t]{2}{*}{ Escala n. } & 3 & 4 & 4 & 2 semanas \\
\hline & $\begin{array}{l}\text { [121211] Cambio en la } \\
\text { ingesta de alimentos. }\end{array}$ & & 2 & 3 & 3 & 2 semanas \\
\hline \multirow{2}{*}{$\begin{array}{l}\text { [1855] } \\
\text { Conocimient } \\
\text { o: Estilo de } \\
\text { vida } \\
\text { saludable. }\end{array}$} & $\begin{array}{l}\text { [185505] Importancia del } \\
\text { agua para la correcta } \\
\text { hidratación. }\end{array}$ & \multirow[t]{2}{*}{ Escala u. } & 2 & 3 & 4 & 2 semanas \\
\hline & $\begin{array}{l}\text { [185510] Importancia de } \\
\text { las raciones de alimentos. }\end{array}$ & & 1 & 3 & 3 & 2 semanas \\
\hline \multirow{2}{*}{$\begin{array}{l}\text { [1854] } \\
\text { Conocimient } \\
\text { o: Dieta } \\
\text { Saludable. }\end{array}$} & $\begin{array}{l}\text { [185403] Relación entre } \\
\text { dieta, ejercicio y peso. }\end{array}$ & \multirow[t]{2}{*}{ Escala u. } & 2 & 4 & 4 & 2 semanas \\
\hline & $\begin{array}{l}\text { [185407] Pautas } \\
\text { nutricionales } \\
\text { recomendadas. }\end{array}$ & & 2 & 3 & 4 & 2 semanas \\
\hline
\end{tabular}

